ISSN 2237-258X

\title{
INDÍGENAS EN ESCUELAS DE EDUCACIÓN BÁSICA DE LA CIUDAD DE MÉXICO
}

\author{
Indigenous people in the basic education schools of Mexico City
}

Indigenas nas escolas de educação básica da Cidade do México

Nicanor Rebolledo*

\section{Resumen}

En este artículo se analiza el doble efecto que provoca la práctica intercultural en escuelas de educación básica en la Ciudad de México, donde son atendidos los niños y niñas de origen indígena. Donde, por un lado, esta práctica elabora alternativas para la implementación de la justicia escolar y, así, permite que los alumnos indígenas participen en las decisiones de las escuelas y, por otro, segrega y, consecuentemente, refuerza la exclusión de los alumnos. El análisis parte del supuesto de que la práctica intercultural promovida por el EIB desencadena un complejo proceso de: a) reconocimiento de la identidad indígena y no indígena; b) la reinvención de la identidad indígena de los alumnos; y c) la construcción de una pedagogía de las culturas indígenas. Es el resultado de una investigación colaborativa realizada en 15 escuelas de educación básica en la Ciudad de México, cuyo objetivo principal es promover el bilingüismo de estudiantes indígenas y la interculturalidad. La reflexión me lleva a concluir que el enfoque de educación intercultural bilingüe promovido por el PEIBDF en estas escuelas, instituye políticas de inclusión y, paradójicamente, alienta la segregación étnica.

PALABRAS CLAVE: Educación. Indígenas. Inclusión.

\begin{abstract}
This article analyzes the double effect caused by intercultural practice in elementary schools in Mexico City, where children of indigenous origin are treated. Where, on the one hand, this practice elaborates alternatives for the implementation of school justice and, thus, allows indigenous students to participate in school decisions and, on the other, segregates and, consequently, reinforces the exclusion of students. The analysis starts from the assumption that the intercultural practice promoted by the EIB triggers a complex process of the a) recognition of indigenous and non-indigenous identity; b) the reinvention of the students' indigenous identity; and c) the construction of a pedagogy of indigenous cultures. It is the result of a collaborative research carried out in 15 elementary schools in Mexico City, whose main objective is to promote bilingualism among indigenous students and interculturality. The reflection leads me to conclude that the approach of bilingual intercultural education promoted by the PEIBDF in these schools, institutes policies of inclusion and, paradoxically, encourages ethnic segregation.
\end{abstract}

KEYWORDS: Education. Indigenous. Inclusion.

\section{Resumo}

* Doctor en Antropología Social por la Universidad Iberoamericana-México. Profesor-investigador de la Universidad
Pedagógica Nacional/México. Investigador Nacional SNI Nivel 2. E-mail: nrebolle@upn.mx.

Revista Educação e Fronteiras On-Line, Dourados/MS, v.8, n.22, p.62-71, jan./abr. 2018 
Neste artigo, analiso o duplo efeito que provoca a prática intercultural nas escolas de educação básica na Cidade do México, onde crianças de origem indígena são assistidas. Por um lado, elabora alternativas para a implementação da justiça escolar e, assim, permite que os alunos indígenas participem das decisões das escolas e, de outro, segrega e, consequentemente, reforça a exclusão dos alunos. A análise parte do pressuposto de que a prática intercultural promovida pelo EIB desencadeia um complexo processo de: a) reconhecimento da identidade indígena e não indígena; b) a reinvenção da identidade indígena dos alunos; e c) a construção de uma pedagogia das culturas indígenas. É o resultado de uma pesquisa colaborativa realizada em 15 escolas de educação básica na Cidade do México, cujo objetivo principal é promover o bilinguismo de estudantes indígenas e a interculturalidade. A reflexão leva-me a concluir que a abordagem de educação intercultural bilíngue promovida pelo PEIBDF nessas escolas institui políticas de inclusão e, paradoxalmente, incentiva a segregação étnica.

PALAVRAS-CHAVE: Educação. Indígenas. Inclusão.

\section{INTRODUCCIÓN}

En este artículo me propongo hacer algunas reflexiones en torno al doble efecto que provoca la práctica intercultural que desarrollan escuelas de educación básica en la Ciudad de México, en las que estudian niños y niñas de origen indígena, así como el futuro que plantea la educación intercultural bilinguie a los infantes indígenas para sus vidas en la gran Ciudad ${ }^{\mathbf{1}}$. Son reflexiones que surgen de investigaciones que hemos venido desarrollando desde el 2003 hasta el momento actual, en 15 escuelas primarias públicas y de los barrios en los que residen estos alumnos, y con las que intentaremos responder provisionalmente a las siguientes interrogantes:

¿La educación intercultural que ofrecen estas escuelas tiende a la segregación indígena y refuerza en consecuencia la exclusión o elabora algunas alternativas de implantación de justicias escolar y permite por este medio posesionar a los indígenas en las decisiones de gobierno de la escuela dentro del marco de derechos que ofrece y abre canales de participación?

Las reflexiones giran en torno a la escolarización "diferenciada" de los estudiantes indígenas y los efectos que puede llegar a tener cuando es asumida como un objetivo y un proceso normalizado de educación indígena y de atención educativa de niños indígenas inscritos en escuelas enclavadas en barrios pobres formados por núcleos de trabajadores informales (vendedores ambulantes, artesanos, albañiles, sirvientas) y de una "alta inseguridad" donde, no sólo aprenden a ser indígenas y se plantean aspiraciones de superación enmarcadas dentro de su propio medio precarizado, muchas veces no para ser mejores y/o cuestionar su situación de pobreza, sino diferentes y en cierta forma para conservar la tradición indígena en la Ciudad. Este tipo de

\footnotetext{
${ }^{1}$ Este trabajo es resultado de una investigación que ha tenido tres fases en su desarrollo, la primera inicio en 2003 con apoyo financiero del CONACYT para desarrollar el Proyecto Bilingüismo, lectocomprensión y comunicación intercultural. El caso de la Escuela Primaria Alberto Correa; la segunda, continua con el Proyecto Bilingüismo y comunicación intercultural. Un estudio sobre la situación sociolingüística de la población indígena del Distrito Federal y de los Estados de Hidalgo y Querétaro, de 2009 con apoyo financiero de la Comisión Nacional para el Desarrollo de los Pueblos Indígenas (CDI); la tercera fase en 2010-2011 comprende el Proyecto Ecología del Bilingüismo y enseñanza del español a estudiantes indígenas" financiado por el CONACYT y la colaboración de un grupo de trabajo integrado por María del Pilar Miguez, Elena Cárdenas, profesoras-investigadoras de la Universidad Pedagógica Nacional. Hemos venido trabajando en 15 escuelas públicas de educación primaria en las que se encuentran estudiando niños de origen indígena de diversas etnias, de un universo de 230 escuelas identificadas con similares características en la Ciudad de México.
} 
educación “diferenciada", intercultural y bilingüe, plantea algunos desafíos y paradójicamente abre una ventana de participación pero también instiga a la segregación.

\section{La investigación colaborativa}

El trabajo colaborativo de investigación que desarrollamos en dichas escuelas no proviene de una simple operación intelectual ni metodológica, sino que es resultado de un trabajo cuyo producto principal es el conocimiento fundamentado de la acción y de una secuencia de acciones (SCHÖN, 1987). Donde la colaboración no es solo un procedimiento facilitador de la colegialidad y el trabajo cooperativo (HARGREAVES, 2003), sino un instrumento para la reflexión y la acción.

Este trabajo lo iniciamos en 2003 en una escuela primaria pública ubicada en la "Zona Rosa", en el Centro de la Ciudad, con niños y niñas hñahñu provenientes del Estado de Querétaro, con el propósito de desarrollar el bilingüismo hñañhu-español; proyecto mediante el cual se buscaba incorporar la enseñanza de la lectura y escritura del hñahñu y la adquisición del español como segunda lengua, así como el fortalecimiento de la identidad y la revitalización del hañhñu.

La información etnográfica recabada a lo largo de los últimos años, así como los datos de campo levantados durante 2008 y 2009 en otras dos escuelas, una localizada en pleno Centro Histórico y otra enclavada un barrio de Xochimilco, ambas con población indígena de diversas etnias, me proporcionó mayores elementos para la reflexión. Además de esto, se suma a dicha información, datos que han sido obtenidos, por una lado, de la aplicación in situ de instrumentos (cuestionarios y guía de entrevistas) a 885 estudiantes indígenas de primero a sexto grado, de diferentes etnias de 15 escuelas ubicadas en la Delegación Iztapalapa, Cuauhtemoc y Gustavo A. Madero y, por otro lado, de la observación etnográfica del ambiente escolar y el desarrollo de actividades escolares de por lo menos ocho escuelas.

Una característica de estas escuelas es la diversidad cultural y lingüística, y la atención específica que reciben desde 2003, fecha en que se instaura el Programa de Educación Intercultural Bilingüe en el Distrito Federal (PEIBDF). La atención específica y "diferenciada" consiste grosso modo en la promoción de un enfoque intercultural y bilingüe para: a) fortalecer la identidad de los estudiantes indígenas y el reconocimiento de las diferencias culturales y lingüísticas; b) reducir la desigualdad y ampliar el acceso de la población a este tipo de educación específica y bilingüe; c) combatir la discriminación y el racismo imperante; c) construir ambientes educacionales en los que se aprenda del Otro y con el Otro, donde el Otro es el indígena y el objetivo es llegar a construir formas de encuentro entre los Otros indígenas (vistos como no citadinos y provenientes de la provincia y de una región indígena) y los no indígenas citadinos; y d) de manera excepcional, son incorporados en la práctica pedagógica contenidos de aprendizaje relacionados con las culturas, la historia, la aritmética, el conteo y las lenguas indígenas.

\section{Indígenas y no indígenas en las escuelas}

Diferenciando a los indígenas en los planteles, es la fase inicial del proceso de reconocimiento y arranque del PEIBDF, y a su vez es el momento crítico en el cual son identificadas algunas dificultades para su implantación, así como los elementos del proceso que ayudan a definir las características que adquiere la diferenciación indígena (interculturalidad) 
dentro de las escuelas y cuando ésta deja de ser parte del proceso para convertirse simplemente en una meta. Hemos podido notar en su fase inicial una afirmación generalizada de que en dichas escuelas no hay indígenas sino tan solo estudiantes. Tal afirmación pudo ser constatada con argumentos de los profesores cuando se les preguntó acerca de la presencia de estudiantes indígenas en sus escuelas, decían, por ejemplo: "aquí no tenemos estudiantes indígenas" o "tenemos estudiantes que vienen de la provincia pero no son indígenas", "sabemos que son indígenas porque no hablan bien español", "nos percatamos que son indígenas porque muestran mayores dificultades para aprender", "notamos que son indígenas porque hablan cortado", etc. Cuando preguntamos directamente a los estudiantes quiénes eran indígenas en los salones las respuestas variaron: "yo no soy indígena, los indígenas son mis padres", "yo soy indígena porque hablo en dialecto", yo hablo la lengua pero no soy indígena" y no faltó quien "delatara" a su compañero señalándolo con el dedo "ese es indígena".

Estas escuelas han experimentado una difícil tarea de identificación y reconocimiento de los estudiantes indígenas. El persistente ocultamiento de la identidad indígena ha dificultado la identificación, así como también el fenómeno de la invisibilidad planeta algunos retos igualmente complejos. De un lado, la declaración de los estudiantes de "el indígena es mi padre (o cualquier ancestro), yo ya no", o "los indígenas son los de mi pueblo, aquí nosotros somos otra cosa diferente", es muy común, y sin duda representa una clara forma de ocultamiento debido al temor a ser discriminado. De otro lado, la declaración de los profesores de "aquí en esta escuela no hay indígenas" o "no quieren descubrirse", son respuestas que pueden ser interpretadas como forma de ocultamiento de una realidad que, piensan, pueden llegar afectar la imagen de la escuela.

El reconocimiento es un concepto abarcador de un cuadro de derechos humanos culturales, lingüísticos, educativos, indígenas, asumidos o negados dentro de las escuelas, que planta algunas dificultades sociológicas y pedagógicas. Nos parecía normal que existieran este tipo de afirmaciones y formas de ocultamiento de la identidad indígena, pues sabíamos que se trataban de formas ideológicas de homogeneización cultural, mediante las cuales se ha podido reproducir la cultura escolar. Sin embargo, frente a esta vieja ideología de homogeneización cultural aún prevaleciente en las escuelas aparece en paralelo el pluralismo cultural como política afirmativa, según la cual existe un derecho a preservar las tradiciones culturales indígenas y de que hay para los indígenas el derecho a vivir de acuerdo con sus propias tradiciones culturales y de usar sus propias lenguas maternas en la educación.

En las últimas décadas observamos una tendencia educativa que busca compatibilizar estos derechos específicos de los indígenas con los derechos universales, incorporando el planteamiento de que existen tradiciones culturales opresivas y que los derechos humanos universales, según Kymlicka (1996), pueden ayudar a combatir esta opresión. La educación intercultural (EIB) a la que nos estamos refiriendo busca en estos casos, no sin tropiezos, compatibilizar las culturas indígenas con la cultura escolar homogeneizadora, los conocimientos indígenas con los conocimientos escolares. En suma, el supuesto general sobre el derecho a la preservación de las tradiciones culturales de los indígenas acarrea algunas dificultades a los educadores, en el sentido de que dificulta las relaciones enriquecedoras entre las culturas en contacto y relación. Al privilegiar la pureza de las tradiciones indígenas frente a la pluralidad, se refuerza el etnocentrismo y la ideología esencialista de unas culturas sobre otras y se crea un ambiente contrario al objetivo de la escuela de impulsar la negociación, la convivencia democrática y la desacralización de las culturas.

En estos casos, el problema radica en que los indígenas que llegan a las escuelas no se convierten automáticamente en alumnos "comunes", en uno de nosotros, como lo hacen los otros 
alumnos "comunes". ¿Pero qué esperamos se produzca en este tipo de escuelas primarias "regulares"? Después de todo no existen escuelas para indígenas, ni escuelas para ciudadanos etiquetados con el término "comunes", sino escuelas oficiales públicas a la que todos tienen libre acceso, donde no hay ningún tipo de restricciones de religión, sexo, lengua o etnia, ni se imponen reglas específicas en su ingreso ni métodos de atención particular. ¿Que deben hacer entonces los profesores no indígenas con los alumnos indígenas que ingresan a las escuelas y que deben hacer para no discriminarlos ni rechazados?.

Con frecuencia, cuando "no hablan bien español" son considerados por sus propios profesores como indígenas y son señalados como los más rezagados de los salones, en muchos de los casos observados, son turnados con la profesora de la Unidad de Servicios de Apoyo a la Educación Regular (Usaer). Como son muy "retraídos" buscan estimularlos a través de actividades culturales que, sabemos, son propias de sus regiones, como los cantos, bailes y cuentos; para estas ocasiones les piden vistan sus trajes regionales y qué hablen algo sobre sus culturas de origen. Con esta forma de trabajo pedagógico los profesores pretenden afirmar lo particular de la cultura de cada estudiante y buscan reforzar la identidad indígena. En este caso el punto de partida de la educación intercultural no pude ser comprender al Otro, sino el reconocimiento de que el Otro es diferente y no comprensible.

En realidad el verdadero problema comienza cuando los profesores aceptan que los indígenas sean "diferentes" y reconozcan algo en ellos que impide sean tratados igual que a sus compañeros de salón, que suelen no hablar "bien" español y académicamente no rinden igual, o sea, los profesores aceptan la diferencia pero la matizan a partir de las desventajas académicas de los alumnos indígenas. Es decir, queda ese vago sentimiento de que los alumnos indígenas no se integran a la dinámica de las clases como el resto de los alumnos. En ocasiones ese sentimiento es matizado con ayudas académicas para atenuar las desventajas y con medidas de asistencia individualizada o con decisiones que terminan por convertirse en actos de separación y distanciamiento físico. Es como si los profesores tuviesen la convicción de que los estudiantes indígenas son realmente incapaces de integrarse a la escuela que los recibe y que su condición étnica no les ayuda a desenvolverse académicamente bien.

En resumen, diferenciando a los indígenas en las escuelas es una típica acción pedagógica que termina por crear condiciones (estereotipos) para el distanciamiento (físico) de los estudiantes indígenas con respecto de los no indígenas, tanto dentro del contexto escolar como fuera de él. La acción pedagógica entendida como imposición y "violencia simbólica impuesta, de una arbitrariedad cultural” (BOURDIEU y PASSERON, 1996, p. 45) que, sin proponérsela, termina convirtiéndose en una acción pedagógica que segrega y sobre todo cuando en el transcurso las escuelas se van transformando en "sitios" exclusivos de indígenas.

\section{Reinventando las identidasdes indígenas}

La reinvención de las culturas indígenas es una típica acción pedagógica que busca reivindicar a las culturas indígenas exaltando el pasado y petrificando el presente. Es un fenómeno que podemos observar a través de las actividades que realizan los profesores con el interés de enseñar "las culturas indígenas" y cuando éstas constituyen contenidos esenciales de aprendizaje. Los profesores argumentan, por ejemplo, no saber qué hacer ante la exigencia de aplicar el enfoque intercultural, sin embargo, realizan lo que entienden que tiene que ver con acciones la integración 
positiva de los estudiantes indígenas a las actividades académicas y culturales de los planteles. Entienden que la interculturalidad es un tema que se relaciona con la presencia de estudiantes indígenas y por eso buscan formas de trabajo que haga posible una integración considerada benéfica, como la atención especial que les brindan a través de la Usaer y la realización de actividades culturales consideradas de igual modo "especiales" (MIGUEZ, 2007).

Mediante la realización de este tipo de actividades "especiales", los profesores tratan de "recrear" las culturas indígenas y de ese modo le otorgan un valor positivo a lo considerado indígena. En este sentido, podríamos decir que los profesores junto con los estudiantes están reinventando las culturas indígenas y produciendo un marco escolar de cultura diferente. Wagner (2010) definió este fenómeno de la invención de la cultura como una práctica humana específica y donde la invención es también una cultura. Esta cultura reinventada que los profesores instrumentalizan con la enseñanza, tiene la peculiaridad de haber sido convertida en un contenido de aprendizaje escolar para ser transmitido. Es decir, en vez de enseñar a través de la cultura o intraculturalmente, reinventan las culturas indígenas a través de lo que los críticos de la educación intercultural llaman actividades de folklorización o por medio de performances construidos a base de diseños ajenos a las culturas de origen. Como veremos, esta perspectiva de creatividad docente está asociada más con la cultura escolar, y los conceptos de cultura que manejan constituyen un legado de la tradición escolar y una forma de reinvención de la tradición escolar.

Además de la creatividad docente como forma de reinvención de las culturas indígenas, podemos incluir también la enorme influencia que han tenido los conceptos de cultura manejados por los antropólogos y los historiadores. Hoy día, por ejemplo, cuando los profesores hablan de cultura inmediatamente la asocian con el pasado indígena y con la historia, así como con la "reminiscencia de las culturas indígenas" y la riqueza que representan para la cultura nacional. Así que el hecho de que en las escuelas haya estudiantes de origen indígena es como un estado de "excepción" para las escuelas de la Ciudad. Continúan empleando el concepto de cultura para destacar las particularidades de la cultura indígena y la diferencia con respecto a la cultura nacional y universal, incluso para referirse a la cultura local (REBOLLEDO, 2007).

Esta idea de cultura difundida por la escuela es básicamente la forma no genética de trasmisión de una comunidad continua, donde la comunidad es entendida como aquella entidad que comparte la misma cultura. De acuerdo con esto, la cultura es entonces aquello que comparte una población y la que la transforma en comunidad. Cultura y comunidad se definen en términos de una relación mutua: cultura es aquello que una población comparte y que la transforma en comunidad. "La comunidad es una subpoblación de una especie, que comparte los rasgos genéticamente trasmitidos, pero que se distingue de la población más amplia por algunas características adicionales: estas, de una u de otra manera dependen de lo que la comunidad, o subpoblación, hace, y no de su equipamiento genético (GELNER, 1989, p. 56).

Las escuelas son los lugares donde se trasmite este concepto particular de cultura indígena opuesto, naturalmente a la cultura nacional y universal (REBOLLEDO, 2004). Transmite discursos tales como el de nación, comunidad, cultura global y lengua común. Los materiales escolares y especialmente los libros de texto gratuitos, inculcan nociones negativas acerca de las lenguas indígenas y nociones positivas de la cultura nacional y universal. Son precisamente estos discursos indigenistas los que han permeado la enseñanza y han trasmitido formas de ver la cultura indígena en oposición a la cultura nacional, occidental y universal.

Por tal motivo, cuando los profesores se refieren a la cultura casi siempre sus razonamientos van acompañados de un argumento demostrativo acerca de la existencia de las culturas indígenas particulares y de una presencia autónoma de cultura. Son razonamientos que se convierten en un 
modo de hablar sobre las tradiciones indígenas y donde les resulta difícil cuestionar su existencia. Aún cuando los profesores han tratado de orientar sus actividades hacia el fortalecimiento de la identidad indígena tomando en cuenta algunas de las recomendaciones proporcionadas por las instituciones encargadas de promoverla, no han podido escapar de las viejas conceptualizaciones y moldes evolucionistas acuñados por la antropología mexicana desde muy entrado el siglo XX, o bien, también podríamos decir que son muestras de resistencia a la innovación o signos de rechazo a las políticas culturales.

Siguen prevaleciendo estas nociones tradicionales de cultura de los profesores, aumentadas y corregidas, reinventadas y actualizadas, que se presentan con la "marca registrada" de las escuelas. Las prácticas culturales que aquí denominamos tradicionales de las escuelas son bastante recientes, a menudo son producto de intercambios culturales y en ocasiones incluso resultan de la invención escolar en su afán de cumplir con el objetivo que plantea la inclusión y la enseñanza intercultural. Junto a estas nociones de cultura que parecen ingenuidades antropológicas de los profesores, proliferaron distintas terminologías institucionales, que cuestionan las nociones tradicionales de cultura indígena "reinventada", no tanto por su acento inofensivo, sino por la asociación directa que suelen hacer sus críticos con el indigenismo alambicado, reaparecido bajo esas nuevas elaboraciones.

\section{Pedagogía de las culturas indígenas}

La pedagogía de las culturas indígenas es otra de las formas típicas de acción pedagógica de las escuelas en cuestión, que comprende no solo la actividad de enseñanza de la cultura indígena, sino también de la enseñanza partiendo de la cultura indígena, o sea, utilizando un marco de enseñanza y aprendizaje indígena, epistemológicamente aún no definido. Un ejemplo de esta tentativa, pudimos apreciarla en una escuela de Xochimilco, con estudiantes mazatecos, nahuas y purépechas, donde no solo fuimos observadores del proceso, sino también participes junto con los profesores del diseño de algunas actividades, tanto culturales como académicas, como la realización de festivales periódicos de las culturas indígenas en la escuela, así como un taller de enseñanza de matemáticas (sistemas de conteo) y otro de lectura y escritura de mazateco.

Previo a los talleres hubo reflexiones de parte de los profesores sobre las posibilidades de ir más allá de estas actividades culturales, por ejemplo, plantearon que los estudiantes mazatecos, nahuas y purépechas aprendan a leer y escribir en sus respectivos idiomas, que aprendan matemáticas, historia de sus pueblos colonizados, sus tradiciones culturales. Siendo conscientes de sus limitaciones al no poder ellos mismo enseñar a leer y escribir en los idiomas indígenas, hicieron sin embargo algunas propuestas de trabajo factibles, como el diseñar una estrategia de trabajo en la que los estudiantes de los grados superiores ayudaran a sus profesores a construir propuestas sencillas de aprendizaje de la lectura y escritura, básicamente del mazateco y el purépecha, dirigidas a enseñar a sus compañeros de primer grado. A esta propuesta de trabajo, se suman dos estudiantes mazatecos de la Licenciatura en Educación Indígena de la Universidad Pedagógica Nacional, para realizar un taller de matemáticas (enseñar propiamente la numeración mazateca), un taller de escritura del mazateco y una actividad que denominaron "hora de conversación en mazateco" y un taller de actividades artísticas (canto del himno nacional en mazateco y canciones mazatecas). 
En los honores a la bandera de los lunes cantan el himno nacional en mazateco y en la medida de los posible lo hacen en los otros idiomas. Con el apoyo de profesores hablantes del mazateco la escuela realizó un curso de tres ciclos escolares de duración, en el que se incluía la enseñanza de la lectura y escritura del mazateco, la enseñanza de la numeración mazateca, cantos y poesía mazateca. ${ }^{2}$

Esto que observamos tiene las características de un proceso de pedagogización de las culturas indígenas, en tanto que presenta una serie de objetivos de enseñanza centrados en las culturas indígenas y las posibilidades que ofrecen las culturas para aprender de ellas. En estos casos los estudiantes aprenden a valorar al indígena y las posibilidades que tiene la propuesta pedagógica de aprender algo nuevo, no solo del indígena, sino de sus culturas y lenguas.

Ante la falta de respuestas concretas de orden metodológico, los profesores han emprendido actividades consideradas por ellos mismos como necesarias para una práctica intercultural activa (como celebraciones en festivales, muestras etnográficas, periódicos murales, danza, poesía y música indígenas, y en algunos casos la introducción del himno nacional en lenguas indígenas en los honores a la bandera los días lunes), cayendo casi inevitablemente en algunas prácticas consideradas por los críticos de la educación intercultural funcional como prácticas escencializadas de cultura indígena.

\section{CONSIDERACIONES FINALES}

La práctica intercultural del programa de EIB, en términos de un proceso concretos, tiene diferentes fases: inicia con el reconocimiento de las particularidades culturales y lingüísticas de los alumnos (etapa de visibilización), continúa con la construcción de la diferencia cultural interna a partir de la reinvención de la identidad indígena y concluye con una práctica intercultural centrada en la pedagogía de la culturas indígenas. Cuando la diferencia cultural es asumida como un objetivo y un proceso normalizado de educación indígena, este proceso termina por segregar a los alumnos indígenas.

En suma, la práctica intercultural, al centrarse en las culturas de origen y en las relaciones entre culturas, promueve el relativismo cultural. La principal crítica a esta postura es que en lugar de promover el relativismo cultural, las tareas deben promover el reconocimiento de la relatividad y la desacralización de todas las culturas. En este sentid, la educación intercultural crítica no debe centrarse en las culturas de origen, ni en las relaciones entre culturas, sino que debe partir de la versión cultural particular de cada individuo para evitar la creación de estereotipos y trabajar sobre la realidad grupal.

Debe estimular el conocimiento de la multiculturalidad presente en la escuela y la sociedad y promover "la comprensión" de múltiples culturas" (BESALÚ, 2002, p. 71). En efecto el valor de la diversidad en el seno de las escuelas es que crea más opciones para cada uno de los alumnos y amplía su ámbito de elección. No obstante, los indígenas en las escuelas no amplían en el mismo sentido el ámbito de elección como lo hacen los miembros de la mayoría que ven recrear sus propios valores sin restricciones.

Por último, para las mayorías de cultura dominante no significa lo mismo abandonar su cultura que cambiar dentro de su propia cultura o que los miembros de las mayorías opten por

\footnotetext{
${ }^{2}$ Durante tres ciclos escolares dos estudiantes mazatecos de la Licenciatura en Educación Indígena de la Universidad Pedagógica Nacional, apoyaron a esta escuela primaria en una serie de actividades, dieron un curso de lectura y escritura del mazateco y de numeración del mazateco, incluía canciones y poesía mazateca.
}

Revista Educação e Fronteiras On-Line, Dourados/MS, v.8, n.22, p.62-71, jan./abr. 2018 
asimilarse a una cultura indígena minoritaria. Para los estudiantes indígenas, sin embargo, cambiar de cultura tiene una significado completamente diferente, implica abandonar su cultura asimilándose a la mayoritaria dominante. Una de las razones básicas de valorar la diversidad cultural en la escuela no significa proponer una educación para los culturalmente diferentes (para indígenas), sino una educación intercultural que haga posible el reconocimiento de la diversidad y la particularidad.

\section{REFERENCIAS}

BESALÚ, X. Diversidad y educación. Madrid: Editorial Síntesis, 2002. (Colleción Teoría e Historia de la Educación.)

BOURDIEU, P. y PASSERON, C. La reproducción. Elementos para una teoría del sistema de enseñanza. México: Fontamara, 1996.

GELNER, E. Cultura, identidad y política. El nacionalismo y los nuevos cambios sociales. Barcelona: Gedisa, 1989.

HARGREAVES, A. Profesorado, cultura y posmodernidad (cambian los tiempos, cambia el profesorado).Madrid: Moratta, 2003.

KYMLICKA, W. Ciudadanía multicultural: una teoría liberal de los derechos de las minorías. Barcelona: Paidós, 1996.

KYMLICKA, W. La política vernácula. Nacionalismo, multiculturalismo y ciudadanía. Barcelona: Paidós, 2003.

MIGUEZ, M.P. Nunca es tarde para comenzar. Bilingüismo indígena y enseñanza bilingüe en primaria. En: Teresinha Bertussi y G. González (Coord.). Anuario educativo mexicano. México: UPN/Porrúa Hermanos, 2007. p. 95-115.

REBOLLEDO, N. Antropología y educación intercultural. Perspectivas interdisciplinarias. En: Mechthild Rutsch y Mette Wacher (Coord.). Alarifes, Amanuenses y Evangelistas. Tradiciones, personajes, comunidades y narrativas de la ciencia en México. México: Instituto Nacional de Antropología e Historia/Universidad Iberoamericana, 2004. p. 397-418. (Colección Científica, Serie Antropología.) 
REBOLLEDO, N. Educación interrumpida. Un caso de migración y bilingüismo en la ciudad de México. México: Ediciones de la Universidad Pedagógica Nacional, 2007. (Colección Más Textos.)

SCHÖN, D. La formación de profesionales reflexivos. Hacia un nuevo diseño de la enseñanza y el aprendizaje de las profesiones. Barcelona: Paidós, 1987.

WAGNER, R. A invenção da cultura. São Paulo: Cosac Naify, 2010.

Recebido em: 20/10/2017

Aceito em: 28/01/2018 